\title{
ON FACTORIZATIONS OF FINITE ABELIAN GROUPS WHICH ADMIT REPLACEMENT OF A Z-SET BY A SUBGROUP
}

\author{
EVELYN E. OBAID \\ Department of Mathematics and Computer Science \\ San Jose State University \\ San Jose, CA 95192 \\ (Received October 20, 1987)
}

ABSTRACT. A subset $A$ of a finite additive abelian group $G$ is a $Z$-set if for a 11 $a \in A$, na $\in A$ for all $n \in Z$.

The purpose of this paper is to prove that for a special class of finite abelian groups, whenever the factorization $G=A \oplus B$, where $A$ and $B$ are Z-sets, arises from the series $G=K_{1} \supset K_{2} \supset \ldots \supset K_{n} \supset\langle 0\rangle$ then there exist subgroups $S$ and $T$ such that the factorization $G=S \oplus T$ also arises from this series. This result is obtained through the introduction of two new concepts: a series admits replacement and the extendability of a subgroup. A generalization of a result of $L$. Fuchs is given which enables establishment of a necessary and sufficient condition for extendability. This condition is used to show that certain series for finite abelian p-groups admit replacement.

KEY WORDS AND PHRASES. Finite abelian group, factorization, Z-set. 1980 AMS SUBJECT CLASSIFICATION CODES. 20K01, $20 \mathrm{~K} 25$.

1. INTRODUCTION.

Let $G$ be a finite abelian additive group and let $A$ and $B$ be subsecs of $G$. If every element $g \in G$ can be uniquely represented in the form $g=a+b$, where $a \in A$, $b \in B$, then we write $G=A \oplus B$ and call this a factorization of $G$. A subset $A$ is said to be a Z-set if for all $a \in A$, na $\in A$ for all $n \in Z$.

A.D. Sands [1] gave a method which yields all factorizations of a finite abelian good group. His method corrects one given previously by G. Hajos [2].

Our main purpose is to prove that for a special class of finite abelian groups, whenever the factorization $G=A \oplus B$, where $A$ and $B$ are Z-sets, arises from the series $\mathrm{G}=\mathrm{K}_{1} \supset \mathrm{K}_{2} \supset \ldots \supset \mathrm{K}_{\mathrm{n}} \supset \mathrm{K}_{\mathrm{n}} \supset \mathrm{K}_{\mathrm{n}} \supset<0>$ (see [3]), then there exist subgroups $\mathrm{S}$ and $\mathrm{T}$ such that factorization $\mathrm{G}=\mathrm{S} \oplus \mathrm{T}$ also arises from this series.

In order to achieve this result we introduce two new concepts: a series admits replacement and the extendability of a subgroup. We prove a generalization of a result of L. Fuchs [4] which enables us to derive a necessary and sufficient condition for extendability. This condition is used to show that certain series for finite abelian $\mathrm{p}$-groups admit replacement. 


\section{PRELIMINARIES.}

We shall use the term "Z-factorization" when referring to a factorization of the form $G=A \oplus B$, where $A$ and $B$ are Z-sets.

Our first two lemmas can be readily verified.

LEMMA 1. Let $G=S \oplus A$, where $S$ is a subgroup of $G$ and $A$ is a $Z$-set. If $H$ and $K$ are subgroups of $G$ with $H=H_{S} \oplus H_{A}, K=K_{S} \oplus K_{A}$, where $H_{S}, K_{S}$ are subgroups of $S$, and $H_{A}, K_{A}$ are Z-sets such that $H_{A} \subseteq A, K_{A} \subseteq A$, then $H \cap K=\left(H_{S} \cap K_{S}\right) \oplus\left(H_{A} \cap K_{A}\right)$.

LEMMA 2. Let $G=A \oplus B$ be a $Z$-factorization of $G$. If $H$ is a subgroup of $G$ such that $A \subseteq H$ then $H=A \oplus(H \cap B)$.

LEMMA 3. Let $G=G^{(0)}=S \oplus A \supset G^{(1)}=S^{(1)} \oplus A^{(1)} \supset \ldots G^{(n)}=S^{(n)} \oplus A^{(n)} \supset$ $G^{(n+1)}=\langle 0\rangle$ be a series for $G$ with $S^{(0)}=s^{(1)} \supset \ldots \supset S^{(n)} \supset\langle 0\rangle$ subgroups of $G$ and $A=A^{(0)} \supset A^{(1)} \supset \ldots \supset A^{(n)} \supset\{0\}$ Z-sets. There exists a refinement of $(2.1)$ which is a composition series for $G$ and the subgroups in this refinement have the same properties as (2.1), i.e. any subgroup, $H$, in the refinement has the form $H=H_{S} \oplus H_{A}$, where $H_{S}$ is a subgroup of $S$ and $H_{A}$ is a z-set, $H_{A} \subseteq A$, and $H \subset K$ implies $H_{S} \subseteq K_{S}$ and $H_{A} \subseteq K_{A}$. Furthermore, if $H \subset K$ are successive groups in the refinement then either $H_{S}=K_{S}$ or $\mathrm{H}_{\mathrm{A}}=\mathrm{K}_{\mathrm{A}}$.

PROOF. It suffices to show that if there exists $\hat{G} \subset G$ with $G^{(i)} \supset \hat{G} \supset G^{(i+1)}$ then $\hat{G}=\hat{S} \oplus \hat{A}$ with $S^{(i)} \supseteq \hat{S} \supseteq S^{(i+1)}, A^{(i)} \supseteq \hat{A} \supseteq A^{(+1)}, \hat{A}$ a Z-set, and either $\hat{S}=S^{(i)}$ or $\hat{A}=A^{(i)}, 0 \leq i \leq n$.

Consider $G^{(i)}=S^{(i)} \oplus A^{(i)} \supset G^{(i+1)}=S^{(i+1)} \oplus A^{(i+1)}, 0 \leq i \leq n$.

Case 1. Suppose that $A^{(i)}=A^{(i+1)}$. Then for any $\hat{G}$ such that $G^{(i)} \supset \hat{G} \supset G^{(i+1)}$ we have $\hat{G}=A^{(i)} \oplus\left(\hat{G} \cap S^{(i)}\right)$ by Lemma 2 . Clearly $S^{(i)} \supset \hat{G} \cap S^{(i)} \supset S^{(i+1)}$. In this case we have $\hat{A}=\hat{A}^{(i)}$.

Case 2. Suppose that $A^{(i)} \neq A^{(i+1)}$. We can insert the subgroup $G=\tilde{G}^{(i+1)}+$ $S^{(i)}=S^{(i)} \oplus A^{(i+1)}$ without altering the structure of the series, $i . e$. we have $G^{(i)}=S^{(i)} \oplus A^{(i)} \supset \tilde{G}=S^{(i)} \oplus A^{(i+1)} \supset G^{(i+1)}=S^{(i+1)} \oplus A^{(i+1)}$.

Let $\hat{G}$ be such that $G^{(i)} \supset \hat{G} \supset G^{(i+1)}$. If $\hat{G}=\tilde{G}$ we are done. If $\tilde{G} \supset \hat{G} \supset G^{(i+1)}$ then by Case $1 \hat{G}$ has the required form. Finally, if $G^{(i)} \supset \hat{G} \supset \tilde{G}$ then by Lemma 2 , $\hat{G}=S^{(i)} \oplus\left(\hat{G} \cap A^{(i)}\right)$. Clearly $A^{(i)} \supset \hat{G} \cap A^{(i)} \supset A^{(i+1)}$. In this case we have $\hat{\mathrm{S}}=\mathrm{S}^{(i)}$. This completes the proof.

THEOREM 1. [5] If $G=B^{(1)} \oplus \ldots \oplus B^{(k)}$, where each $B^{(i)}$ is a $Z$-set, $1 \leq i \leq k$, and if $G=N^{(1)} \oplus \ldots \oplus N^{(r)}$, where each $N^{(j)}$ is a subgroup of $G, 1 \leq j \leq r$, such that $\left(\left|N^{(i)}\right|,\left|N^{(j)}\right|\right)=1$ for $i \neq j$, then

(a) $B^{(i)}=\left(N^{(1)} \cap B^{(i)}\right) \oplus \ldots \oplus\left(N^{(r)} \cap B^{(i)}\right), 1 \leq i \leq k$, and

(b) $N^{(j)}=\left(N^{(j)} \cap B^{(1)} \oplus \ldots \oplus\left(N^{(j)} \cap B^{(k)}\right), 1 \leqq j \leqq r\right.$. 
The following lemma is a direct consequence of the Second Isomorphism Theorem.

LEMMA 4. Let $U, U_{1}$, and $K$ be subgroups of $G$ with $U_{1} \subseteq U$. Then [U $\left.\cap K: U_{1} \cap K\right] \leq$ [U: $\left.\mathrm{U}_{1}\right]$.

Let $S$ be a subgroup of $G$. We will say that $S$ is homogeneous if $S$ is a direct sum of cyclic groups of the same order.

Theorem 2, which is a generalization of the following result of L. Fuchs [4], p.79), can be readily verified.

(Fuchs) Let $\mathrm{S}$ be a pure homogeneous subgroup of $\mathrm{G}$ of exponent $\mathrm{p}^{\mathrm{k}}$ and let $\mathrm{H}$ be a subgroup of $G$ satisfying $\mathrm{p}^{k} G \subseteq H$ and $S \cap H=\langle 0\rangle$. If $M$ is a subgroup of $G$ maximal with respect to the properties $H \subseteq M$ and $M \cap S=\langle 0\rangle$ then $G=S \oplus M$.

THEOREM 2. Let $S=\underset{i=1}{n} S_{i}$ be a pure subgroup of $G$ with $S_{i}, 1 \leq i \leq n$, homogeneous of exponent $p^{k_{i}}, k_{1}>k_{2} \ldots>k_{n}$, and let $U \subseteq G$. There exists a subgroup, $T$, of $G$ with $U \subseteq T$ and $G=S \oplus T$ if and only if $\left[p^{k_{j}} j_{G}+\underset{i>j}{\oplus} S_{i}+U\right] \cap S_{j}=\langle 0\rangle, 1 \leq j \leq n$.

3. REDUCTION TO THE CAUSE OF P-GROUPS.

Consider the series

$$
\left.G=G^{(0)}=S \oplus A \supset G^{(1)}=S^{(1)} \oplus A^{(1)} \supset \ldots G^{(n)}=S^{(n)} \oplus A^{(n)} \supset<0\right\rangle
$$

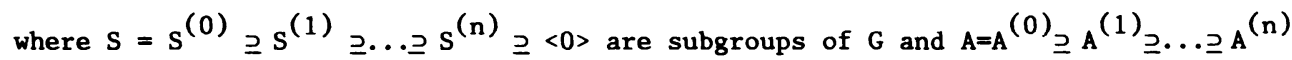
$\supseteq\{0\}$ are $\mathrm{Z}$-sets. We say the series (3 1 ) admits replacements if there exist sub-

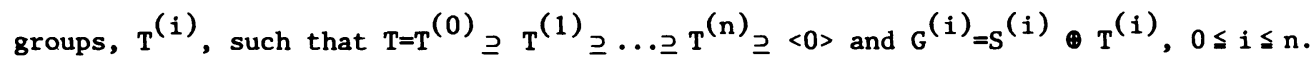
Let us note that by Proposition 1 [3] there exist subgroups $T^{(i)}$ such that $G^{(i)}=S^{(i)} \oplus T^{(i)}, 0 \leq i \leq n$. However it is not necessarily the case that $T^{(0)} \supseteq$ $T^{(1)} \supseteq \ldots \supseteq T^{(n)}$. This problem will be treated in the next section.

A group C admits replacement if every series for $G$ of the form (3.1) admits replacements. The following theorem enables us to restrict our investigations in this area to the case of $\mathrm{p}$-groups.

THEOREM 3. Let $G=\oplus G_{p}$, where the $G_{p}$ are the primary components of $G$. $G$ admits replacement if and only if for each $P, G_{p}$ admits replacement.

PROOF. Suppose $G$ admits replacement. Let $H=G_{p}$ for some $P$ and let $H=H^{(0)}=S^{(0)} \oplus A^{(0)} \supset H^{(1)}=S^{(1)} \oplus A^{(1)} \supset \ldots H^{(m)}=S^{(m)} \oplus A^{(m)} \supset\langle 0\rangle$ be a series for $H$ with $S^{(0)} \supseteq S^{(1)} \supseteq \ldots \supseteq S^{(m)} \supset<0>$ subgroups of $H$ and $A^{(0)} \supseteq A^{(1)} \supseteq \ldots \supseteq A^{(m)} \supset\{0\}$ Z-sets. Define $K=\underset{p^{\prime} \neq p_{p^{\prime}}}{ } G$ so that $G=H \oplus K=\left(S^{(0)} \oplus K\right)$ $\oplus A^{(0)}$. Then $G=G^{(0)}=\left(S^{(0)} \oplus K\right) \oplus A^{(0)} \supset H^{(0)}=S^{(0)} \oplus A^{(0)} \supset H^{(1)}=S^{(1)} \oplus A^{(1)}$ $\supseteq \mathrm{H}^{(\mathrm{m})}=\mathrm{S}^{(\mathrm{m})} \oplus \mathrm{A}^{(\mathrm{m})} \supseteq\langle 0\rangle$ is a series for $\mathrm{G}$ which by hypothesis admits replacements. Consequently the series $H=H^{(0)} \supset H^{(1)} \supset \ldots \supset H^{(m)} \supset<0>$ admits replacements. 
Conversely, suppose $G_{p}$ admits replacement for each $p$. Let $G=G^{(0)}=S^{(0)} \oplus A^{(0)} \supset G^{(1)}=S^{(1)} \oplus A^{(1)} \supset \ldots G^{(n)}=S^{(n)} \oplus A^{(n)} \supset\langle 0\rangle$ be a series for $G$ with $S^{(0)} \supseteq S^{(1)} \supseteq \ldots \supseteq S^{(n)} \supseteq\langle 0\rangle$ subgroups of $G$ and $A(0) \supseteq A^{(1)}$ $\supseteq \ldots A^{(n)} \supseteq\{0\}$ Z-sets. For each $p$ define $G_{p}^{(i)}$ to be the p-primary component of $G^{(i)}, 0 \leq i \leq n$, so that $G_{p}^{(i)}=G_{p} \cap G^{(i)}$ and $G^{(i)}=\underset{p}{G} G_{p}^{(i)}$. By Theorem 1 we have that $G_{p}^{(i)}=\left(S^{(i)} \cap G_{p}^{(i)}\right) \oplus\left(A^{(i)} \cap G_{p}^{(i)}\right), 0 \leq i \leq n$.

Define $s_{p}^{(i)}=s^{(i)} \cap G_{p^{\prime}}^{(i)}, A_{p}^{(i)}=A^{(i)} \cap G_{p}^{(i)}, 0 \leq i \leq n . \quad$ Clearly $s_{p}^{(0)} \supseteq s_{p}^{(1)} \supseteq \ldots \supseteq S_{p}^{(n)} \supseteq\langle 0\rangle$ and $A_{p}^{(0)} \supseteq A_{p}^{(1)} \supseteq \ldots \supseteq A_{p}^{(n)} \supseteq\{0\}$. Thus for each $p$, $\left.G_{p}=G_{p}^{(0)}=S_{p}^{(0)} \oplus A_{P}^{(0)} \supset G_{P}^{(1)}=S_{p}^{(1)} \oplus A_{p}^{(1)} \supset \ldots \supset G_{p}^{(n)}=S_{p}^{(n)} \oplus A_{p}^{(n)} \supset<0\right\rangle$ is a series for $G_{p}$ which by assumption admits replacements. Thus for each $p$ there exist subgroups $T_{p}^{(i)}$ such that

(i) $T_{p}=T_{p}^{(0)} \supseteq T_{p}^{(1)} \supseteq \ldots \supseteq T_{p}^{(n)} \supset<0>$

and

$$
\text { (ii) } G_{p}^{(i)}=S_{p}^{(i)} \bullet T_{p}^{(i)}, 0 \leq i \leq n .
$$

Define $T^{(i)}=\sum_{p} T_{p}^{(i)}, 0 \leq i \leq n$. Note that this sum is direct. From (i) we have that $T=T^{(0)} \supset T^{(1)} \supset \ldots \supset T^{(n)} \supset\langle 0\rangle$ and (ii) implies that

$G^{(i)}=\underset{p}{\oplus} G_{p}^{(i)}=\underset{p}{\oplus}\left(S_{p}^{(i)} \oplus T_{p}^{(i)}\right)=\left(\underset{P}{\oplus S_{p}^{(i)}}\right) \oplus\left(\underset{p}{\oplus} T_{p}^{(i)}\right)=S^{(i)} \oplus T^{(i)}$,

$0 \leq \mathrm{i} \leq \mathrm{n}$. This completes the proof.

\section{EXTENDABILITY}

Let $G=S \oplus A \supset G^{\prime}=S^{\prime} \oplus A^{\prime}=S^{\prime} \oplus T^{\prime}$, where $S^{\prime} \subseteq S$ are subgroups of $F, A^{\prime} \subseteq A$ are $Z$-sets, and $T^{\prime}$ is a subgroup of $G^{\prime}$. We say $T^{\prime}$ is extendable to $G$ if there exists $T$, a subgroup of $G$, such that $T^{\prime} \subseteq T$ and $G=S \oplus T$.

The following theorem provides a necessary and sufficient condition for extendability of a subgroup $T^{\prime}$ when $G$ is a p-group.

THEOREM 4. Let $G$ be a finite abelian $p$-group of exponent $p^{k}$ and let $G^{\prime}$ be a subgroup of $G$. Suppose $G=S \oplus A, G^{\prime}=S^{\prime} \oplus A^{\prime}=S^{\prime} \oplus T^{\prime}$ with $S^{\prime} \subseteq S$, subgroups of $G, A^{\prime} \subseteq A, Z$-sets, and $T^{\prime}$ a subgroup of $G^{\prime}$. $T^{\prime}$ is extendable to $G$ if and only if there exist subgroups, $T_{i}$, such that $T^{\prime} \supseteq T_{1} \supseteq T_{2} \supseteq \ldots \supseteq T_{k-1} \supseteq\langle 0\rangle$ and $G^{\prime} \cap p^{i_{G}}=$ $\left.S^{\prime} \cap p^{i} S\right) \oplus T_{i}, 1 \leqq i \leq k-1$. 
PROOF. By Lemma 1 we have that $G^{\prime} \cap p^{i_{G}}=\left(S^{\prime} \cap p^{i} S\right) \oplus\left(A^{\prime} \cap p^{i} A\right), 1 \leq i \leq k-1$.

Assume there exists subgroups, $T_{i}$; with $T^{\prime} \supseteq T_{1} \supseteq \ldots \supseteq T_{k-1} \supseteq\langle 0\rangle$ and $G^{\prime} \cap p^{i} G=\left(S^{\prime} \cap p^{i} s\right) \oplus T_{i}, 1 \leqq i \leqq k-1$. Let $s=\underset{i=1}{\mathbb{k}} s_{i}$, where each $S_{i}$ is homogeneous of exponent $p^{i}, 1 \leqq i \leqq k$. By Theorem 2, to prove the existence of a subgroup, $T$, of $G$ with $T \supseteq T^{\prime}$ and $G=S \oplus T$ we must verify

$$
\left(p^{k_{G}}+\underset{i<k}{\oplus} S_{i}+T^{\prime}\right) \cap S_{k}=\left(\underset{i<k}{\oplus} S_{i}+T^{\prime}\right) \cap S_{k}=<0>
$$

and

$$
\left(p^{j_{G}}+\underset{i<j}{\oplus} S_{i}+T^{\prime}\right) \cap s_{j}=<0>, 1 \leqq j \leqq k-1
$$

For (4.1), suppose $s_{k}=\underset{i<k}{\sum} s_{i}+t^{\prime}, s_{i} \in s_{i}, 1 \leq i \leq k, t^{\prime} \in T^{\prime}$. Since $T^{\prime} \subseteq G^{\prime}$ we have $t^{\prime}=s^{\prime}+a^{\prime}, s^{\prime} \in S^{\prime}, a^{\prime} \in A^{\prime}$. Thus $s_{k}=\underset{i<k}{\sum} s_{i}+s^{\prime}+a^{\prime}$ so that $a^{\prime}=0$ by the definition of $S \oplus A$. But then $t^{\prime} \in S^{\prime} \cap T^{\prime}=\langle 0\rangle$. Consequently, $s_{k}=\sum_{i<k} s_{i}$. However, since $s$ is a direct sum of $s_{1}, s_{2}, \ldots, s_{k}$, we have $s_{k}=0+0+$ $\ldots+0+s_{k}$. Together these imply that $s_{k}=0$. Hence (4.1) is true.

Let $1 \leqq j<k$. Note that $p^{j} S_{i}=\left\langle 0>\right.$ if $i \leqq j$. Thus we have $p^{j_{G}}=\underset{i>j}{\oplus p^{j} S_{i}} \oplus p^{j} A$.

Suppose

$$
s_{j}=p^{j} g+\sum_{i<j} s_{i}+t^{\prime}=\sum_{i>j} p^{j} s_{i}+p^{j} a+\sum_{i<j} s_{i}+t^{\prime}
$$

where $s_{i} \in S_{i}, 1 \leq i \leq k, a \in A, t^{\prime} \in T^{\prime}$. Since $T^{\prime} \subseteq G^{\prime}=S^{\prime} \oplus A^{\prime}$ we have

$$
t^{\prime}=s^{\prime}+a^{\prime}, s^{\prime} \in S^{\prime}, a^{\prime} \in A^{\prime}
$$

Thus $s_{j}=\sum_{i>j} p^{j} s_{i}+\underset{i<j}{\sum} s_{i}+s^{\prime}+p^{j} a+a^{\prime}$. Therefore

$$
\begin{aligned}
& p^{j} a=-a^{\prime} \in p^{j} \cap \cap A^{\prime} \subseteq G^{\prime} \cap p^{j_{G}} \\
& s_{j}=\sum_{i>j} p^{j} s_{i}+\sum_{i>j} s_{i}+s^{\prime}
\end{aligned}
$$

Since $p^{j_{A}} \cap A^{\prime}$ is clearly a Z-set we have from (4.4) that $a^{\prime} \in p^{j} \cap A^{\prime}$. By hypothesis, $G^{\prime} \cap p^{j_{G}}=\left(S^{\prime} \cap p^{j_{S}}\right) \oplus T_{j}$ with $T_{j} \subseteq T^{\prime}$. Therefore (4.4) implies that $a^{\prime}=\sum_{i>j} p^{j} \tilde{s}_{i}+t_{j}$, where $\underset{i>j}{\sum} p^{j} \tilde{s}_{i} \in S^{\prime} \cap p^{j} S=S^{\prime} \cap \underset{i>j}{\oplus} p^{j} S_{i}$ and $t_{j} \in T_{j} \subseteq T^{\prime}$. But then (4.3) becomes $t^{\prime}=s^{\prime}+\sum_{i>j} p^{j} \tilde{s}_{i}+t_{j}$, where $s^{\prime}+\sum_{i>j} p^{j} \tilde{s}_{i} \in S^{\prime}$ and $t_{j} \in S^{\prime}$ and $t_{j} \in T^{\prime}$. Consequent $1 y$ 
$s^{\prime}+\sum_{i>j} p^{j} \tilde{s}_{i}=0$ by the definition of $s^{\prime} \oplus T^{\prime}$, and we have $s^{\prime}=-\underset{i>j}{\sum} p^{j} \tilde{s}_{i}$

Substituting this expression for $s^{\prime}$ into (4.5) we obtain

$s_{j}=\sum_{i>j} p^{j} s_{i}+\sum_{i>j} s_{i}-\sum_{i>j} p^{j} \tilde{s}_{i}$ so that $s_{j}=0$. Hence (4.2) is true.

Conversely, suppose there exists a subgroup $T \supseteq T^{\prime}$ such that $G=S \oplus T$. By Lemma 1, $G^{\prime} \cap p^{i} G=\left(S^{\prime} \cap p^{i} S\right) \oplus\left(T^{\prime} \cap P^{i_{i}} T\right), 1 \leqq i \leqq k-1$. Clearly $T^{\prime} \supseteq T^{\prime} \cap p^{T} \supseteq T^{\prime} \cap$ $\mathrm{p}^{2} \mathrm{~T} \supset \ldots \supset \mathrm{T}^{\prime} \cap \mathrm{p}^{k-1} \mathrm{~T}$. Thus we can complete the proof by choosing $T_{i}=T^{\prime} \cap \mathrm{p}^{i} \mathrm{~T}$, $1 \leqq i \leqq k-1$.

Let us note that if $G=S \oplus A$, where $S$ is a subgroup of $G$ and $A$ is a $Z$-set, is an elementary abelian $\mathrm{p}^{-g r o u p,}$ and $G^{\prime}$ is a subgroup of $G_{\text {such that }} G^{\prime}=S^{\prime} \oplus A^{\prime}$ $=S^{\prime} \oplus T^{\prime}$, where $S^{\prime} \subseteq S, T^{\prime} \subseteq G^{\prime}$, and $A^{\prime}$ is a $Z$-set contained in $A$, then $T^{\prime}$ is always extendable to $\mathrm{G}$.

LEMMA 5. Let $G=S \oplus A \supseteq G^{\prime}=S^{\prime} \oplus A$ be a series for $G$ with $S^{\prime} \subseteq S$ subgroups of $G$ and $A$ a $Z$-set. If $T$ is a subgroup of $G^{\prime}$ such that $G^{\prime}=S^{\prime} \oplus T$ then $G=S \oplus T$.

PROOF. Let $\tilde{S}$ be a set of coset representatives for $S$ modulo $S^{\prime}$.

Then $G=S \oplus A=\tilde{S} \oplus S^{\prime} \oplus A=\tilde{S} \oplus S^{\prime} \oplus T=S \oplus T$.

5. SOME GROUPS WHICH ADMIT REPLACEMENT.

We noted in Section 4 that given the series

$\left.G=G^{(0)}=S^{(0)} \oplus A^{(0)} \supset G^{(1)}=S^{(1)} \oplus A^{(1)} \supset \ldots G^{(n)}=S^{(n)} \oplus A^{(n)} \supset<0\right\rangle$

where $S=S^{(0)} \supseteq S^{(1)} \supseteq \ldots \supseteq S^{(n)} \supseteq\langle 0\rangle$ are subgroups and $A=A^{(0)} \supseteq A^{(1)} \supseteq \ldots \supseteq A^{(n)}$

$\underline{\supseteq}\{0\}$ are Z-sets, one can always find subgroups $T^{(i)}$ such that $G^{(i)}=S^{(i)} \oplus T^{(i)}$, $0 \leqq i \leqq n$, although it need not be the case that $T=T^{(0)} \supseteq T^{(1)} \supseteq \ldots \supseteq T^{(n)} \supseteq\langle 0\rangle$.

However, by applying the extendability criterion of Theorem 4, we can ensure that for each $i, 0 \leq i \leq n$, our choice of the subgroup $T^{(i)}$ will be extendable to each $G^{(\alpha)}$, $\alpha \leq i$, and consequent1y we will have $T=T^{(0)} \supseteq T^{(1)} \supseteq \ldots \supseteq T^{(n)}$.

We will briefly illustrate how successive applications of Theorem 4 when $G$ is a finite abelian $p$-group of exponent $p^{3}$ results in Figure 1 since this lattice-type structure clarifies the proof of the major theorem in this section. By Lemma 3 we may assume that (5.1) is a composition series for $G$.

We introduce the following notation to simplify the discussion:

$$
\begin{aligned}
& G_{j, l}^{(i)}=G^{(i)} \cap p^{j_{G}}(l), \\
& S_{j, l}^{(i)}=S^{(i)} \cap p^{j_{S}}{ }^{(l)}, \\
& A_{j, l}^{(i)}=A \\
& (i) \cap p^{j_{A}}(l),
\end{aligned}
$$


where $0 \leqq i \leqq n, 0 \leqq l \leqq n, j=1,2$. By Lemma 1 we have $G_{j, l}^{(i)}=s_{j, l}^{(i)} \oplus A_{j, l}^{(i)}$, $0 \leqq i \leqq n, 0 \leqq \ell \leqq n, j=1,2$.

In addition, $T_{j, l}^{(i)}$ will denote any subgroup of $G_{j, l}^{(i)}$ such that $G_{j, l}^{(i)}=s_{j, l}^{(i)} \oplus A_{j, l}^{(i)}=S_{j, l}^{(i)} \oplus T_{j, l}^{(i)}$, and $T_{h, l, l}^{(i)}$ will denote any subgroup of $p^{h_{G}}(i)$ such that $p^{h} G_{1, l}^{(i)}=p^{h} S_{1, l}^{(i)} \oplus p^{h} A_{1, l}^{(i)}=p^{h} S_{1, l}^{(i)} \oplus T_{h, 1, l}^{(i)} 0 \leqq i \leqq n, 0 \leqq l \leqq i, j=1,2, h$ a nonnegative integer.

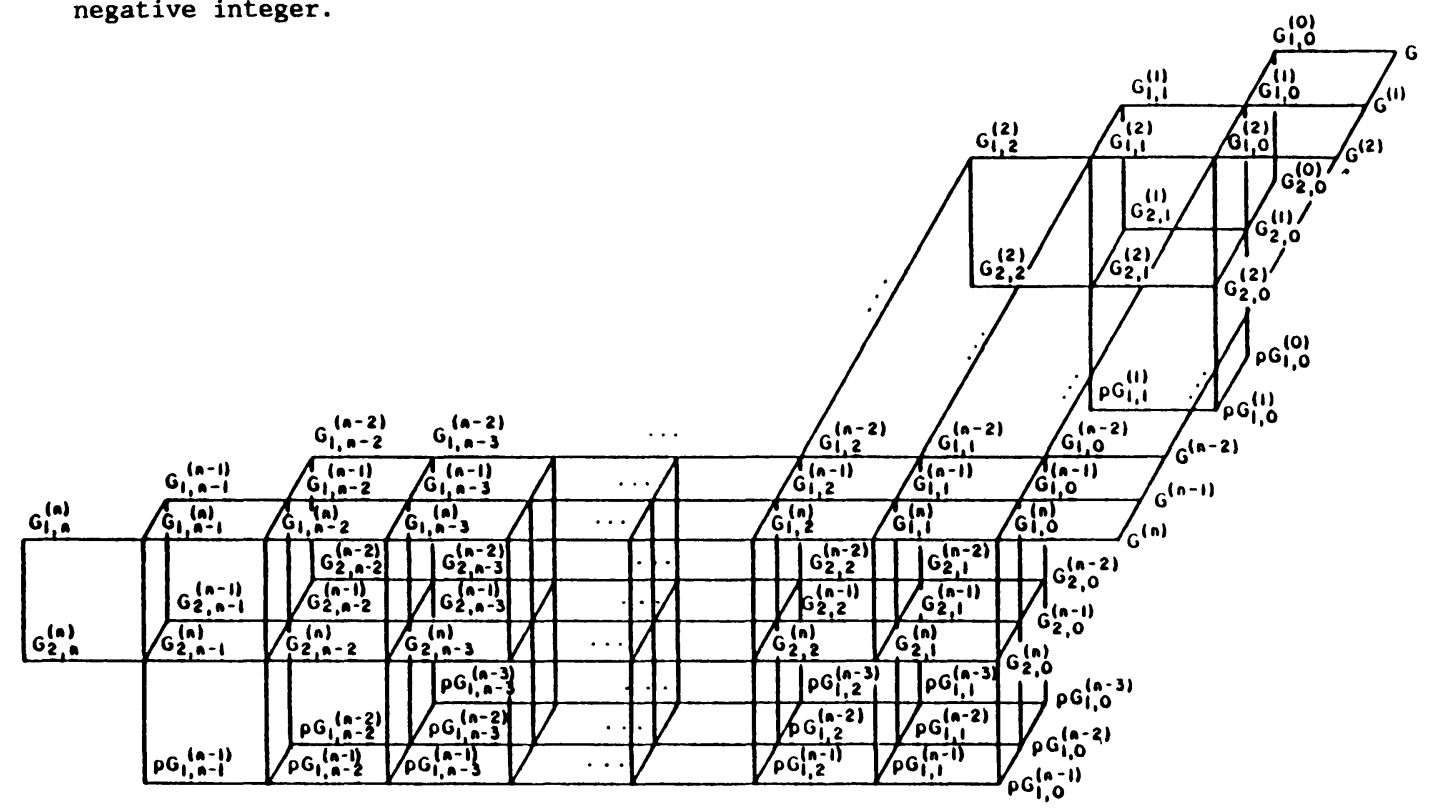

Consider a subgroup $T^{(n)}$ such that in the series $(5.1)$ we have $G^{(n)}=S^{(n)} \oplus T^{(n)}$. By Theorem 4, $T^{(n)}$ will be extendable to $G^{(i)}, 0 \leq i \leq n-1$, if an only if there exist subgroups $T_{j, 1}^{(n)}$ such that $T^{(n)} \supseteq T_{1, i}^{(n)} \supseteq T_{2, i}^{(n)}$ with $G_{j, i}^{(n)}=S_{j, i}^{(n)}, 0 \leq i \leq n-1, j=1,2$. We have the following array for the containments of the subgroups $G_{j, i}^{(n)}, 0 \leq i \leq n, j=1,2$ :

$$
\begin{aligned}
& G_{1, n}^{(n)}=p G^{(n)} \supseteq G_{1, n-1}^{(n)} \supseteq G_{1, n-2}^{(n)} \supseteq \ldots \supseteq G_{1,1}^{(n)} \supseteq G_{1,0}^{(n)} \supseteq G^{(n)} \\
& U 1 \quad U 1 \quad U 1 \quad U 1 \\
& G_{2, n}^{(n)}=p^{2} G^{(n)} \supseteq G_{2, n-1}^{(n)} \supseteq G_{2, n-2}^{(n)} \subset \ldots \subseteq G_{2,1}^{(n)} \supseteq G_{2,0}^{(n)}
\end{aligned}
$$

Thus if we can find subgroups $T_{j, i}^{(n)}, 0 \leqq i \leqq n, j=1,2, T^{(n)}$, such that

$$
\begin{aligned}
& T_{1, n}^{(n)} \subseteq T_{1, n-1}^{(n)} \subseteq T_{1, n-2}^{(n)} \subseteq \ldots \subseteq T_{1,1}^{(n)} \subseteq T_{1,0}^{(n)} \subseteq T^{(n)} \\
& U 1 \quad U 1 \quad U 1 \quad U 1 \\
& T_{2, n}^{(n)} \subseteq T_{2, n-1}^{(n)} \subseteq T_{2, n-2}^{(n)} \subseteq \ldots \subseteq T_{2,1}^{(n)} \subseteq T_{2,0}^{(n)}
\end{aligned}
$$


and $G_{j, i}^{(n)}=S_{j, i}^{(n)} \oplus T_{j, i}^{(n)}, 0 \leqq i \leqq n, j=1,2, G^{(n)}=S^{(n)} \oplus T^{(n)}$, we would have $T^{(n)}$ extendable to $G^{(i)}, 0 \leqq i \leqq n-1$.

Later it will become apparent that we need $T_{1, i}^{(n)}$ extendable to $G_{1, i}^{(n-1)}$,

$0 \leqq i \leq n-1$. We will show how this can be incorporated in our discussion on $T^{(n)}$.

Using Lemma 4 with $U=G^{(n-1)}, U_{1}=G^{(n)}, K=p G^{(i)}, 0 \leqq i \leqq n-1$, we have $\left[G_{1, i}^{(n-1)}: G_{1, i}^{(n)}\right] \leqq p$. Thus $p G_{1, i}^{(n-1)} \subseteq G_{1, i}^{(n)}$ so that $G_{1, i}^{(n)} \cap p G_{1, i}^{(n-1)}=p G_{1, i}^{(n-1)}, 0 \leq i \leq n-1$.

Note that $G_{1, i}^{(n-1)}$ has exponent at most $p^{2}$. By Theorem $4, T_{1, i}^{(n)}$ is extendable to $G_{1, i}^{(n-1)}$, $0 \leqq i \leqq n-1$, if and only if there exists a subgroup $T_{1,1, i}^{(n-1)}$ such that $T_{1, i}^{(n)} \supseteq T_{1,1, i}^{(n-1)}$ and $\mathrm{pG}_{1, i}^{(n-1)}=\mathrm{pS}_{1, i}^{(n-1)} \oplus T_{1,1, i}^{(n-1)}, 0 \leq i \leq n-1$. Since $p G_{1, i}^{(n-1)}=p\left(G^{(n-1)} \cap p G^{(i)}\right)$ $\subseteq p G^{(n-1)} \cap p^{2} G^{(i)} \subseteq G^{(n)} \cap p^{2} G^{(i)}=G_{2, i}^{(n)}, 0 \leqq i \leq n-1$, we have the following array for the subgroups $G^{(n)}, G_{j, i}^{(n)}, p G_{1, i}^{(n-1)}, 0 \leqq i \leqq n-1, j=1,2$ :

$$
\begin{aligned}
& G_{1, n}^{(n)} \subseteq G_{1, n-1}^{(n)} \subseteq G_{1, n-2}^{(n)} \subseteq \ldots \subseteq G_{1,1}^{(n)} \subseteq G_{1,0}^{(n)} \subseteq G^{(n)} \\
& \begin{array}{lllll}
u 1 & \text { U1 } & \text { U1 } & \text { U1 } & \text { U1 }
\end{array} \\
& G_{2, n}^{(n)} \subseteq G_{2, n-1}^{(n)} \subseteq G_{2, n-2}^{(n)} \subseteq \ldots \subseteq G_{2,1}^{(n)} \subseteq G_{2,0}^{(n)} \\
& \begin{array}{llll}
\text { U1 } & \text { U1 } & \text { U1 } & \text { U1 }
\end{array} \\
& \mathrm{pG}_{1, n-1}^{(\mathrm{n}-1)} \subseteq \mathrm{pG}_{1, \mathrm{n}-2}^{(\mathrm{n}-1)} \subseteq \ldots \subseteq \mathrm{pG}_{1,1}^{(\mathrm{n}-1)} \subseteq \mathrm{pG}_{1,0}^{(\mathrm{n}-1)}
\end{aligned}
$$

Thus if we can find subgroups $T_{j, i}^{(n)}, T_{1,1, i}^{(n-1)}, 0 \leq i \leq n, 0 \leq i^{\prime} \leq n-1, j=1,2$, $T^{(n)}$ such that

$$
\begin{aligned}
& T_{1, n}^{(n)} \subseteq T_{1, n-1}^{(n)} \subseteq T_{1, n-2}^{(n)} \subseteq \cdots \subseteq T_{1,1}^{(n)} \subseteq T_{1,0}^{(n)} \subseteq T^{(n)} \\
& \begin{array}{lllll}
\text { U1 } & \text { U1 } & \text { U1 } & \text { U1 } & \text { U1 }
\end{array} \\
& T_{2, n}^{(n)} \subseteq T_{2, n-1}^{(n)} \subseteq T_{2, n-2}^{(n)} \subseteq \cdots \subseteq T_{2,1}^{(n)} \subseteq T_{2,0}^{(n)} \\
& \text { U1 U1 } \quad \text { U1 } \quad \text { U1 } \\
& T_{1,1, n-1}^{(n-1)} \subseteq T_{1,1, n-2}^{(n-1)} \subseteq \cdots \subseteq T_{1,1,1}^{(n-1)} \subseteq T_{1,1,0}^{(n-1)}
\end{aligned}
$$

with $G_{j, i}^{(n)}=s_{j, i}^{(n)} \oplus T_{j, i^{\prime}}^{(n)}, p G_{1, i^{\prime}}^{(n-1)}=p S_{1, i^{\prime}}^{(n-1)} \oplus T_{1,1, i^{\prime}}^{(n-1)} 0 \leq i \leq n, 0 \leq i^{\prime} \leq n-1$, $j=1,2 G^{(n)}=S^{(n)} \oplus T^{(n)}$, we would have $T_{1, i}^{(n)}$ extendable to $G_{1, i}^{(n-1)}, 0 \leq i \leq n-1$, and $T^{(n)}$ extendable to $G^{(i)}, 0 \leqq i \leqq n-1$. In particular, we would know there exists 
a subgroup $T^{(n-1)}$ with $T^{(n-1)} \supseteq T^{(n)}$ and $G^{(n-1)}=S^{(n-1)} \oplus T^{(n-1)}$. However, we must ensure that our choice for $T^{(n-1)}$ is extendable to $G^{(i)}, 0 \leq i \leq n-2$. Appyling the previous argument to $T^{(n-1)}$ and then to $T^{(i)}, 0 \leq i \leq n-3$, we obtain Figure 1. We remark that lattice-type structures similar to Figure 1 can be obtained for finite abelian $p$-groups of exponent $p^{k}$, where $k$ is any non-negative integer. Such structures become rather complicated when the exponent of the group exceeds $p^{3}$.

The following definitions will facilitate references to Figure 1 .

DEFINITION 1. The row for $G^{(i)}, 0 \leq i \leq n$, is the series $G^{(i)} \geq G_{1,0}^{(i)} \supseteq G_{1,1}^{(i)} \supseteq \cdots \geq G_{1, i}^{(i)}$.

DEFINITION 2. The row for $G_{2,0}^{(i)}, 0 \leq i \leq n$, is the series $G_{2,0}^{(i)} \supseteq G_{2,1}^{(i)} \supseteq G_{2,2}^{(i)} \supseteq \ldots \supseteq G_{2, i}^{(i)}$.

DEFINITION 3. The row for $\mathrm{pG}_{1,0}^{(i)}, 0 \leq i \leq n-1$, is the series $\mathrm{pG}_{1,0}^{(i)} \supseteq \mathrm{PG}_{1,1}^{(i)} \supseteq \mathrm{pG}_{1,2}^{(i)} \supseteq \cdots \supseteq \mathrm{pG}_{1, i}^{(i)}$.

DEFINITION 4. The sub-figure for $G^{(i)}, 0 \leq i \leq n$, consists of the rows for $G^{(\ell)}, G_{2,0}^{(\ell)}$, and $\mathrm{pG}_{1,0}^{(\ell)}, i \leq \ell \leq n, i-1 \leq \ell^{\prime} \leq \mathrm{n}-1$.

DEFINITION 5. We say the sub-figure for $G^{(i)}=S^{(i)} \oplus A^{(i)}, 0 \leq i \leq n$, is complete if

(i) for every subgroup $H$ in the sub-figure for $G^{(i)}, H=S_{H} \oplus A_{H}$, $S_{H}$ a subgroup of $S^{(i)}, A_{H}$ a Z-set, $A_{H} \subseteq A^{(i)}$, there exists a subgroup $T_{H}$ such that $\mathrm{H}=\mathrm{S}_{\mathrm{H}} \oplus \mathrm{T}_{\mathrm{H}}$,

(ii) for all sub-groups $H, K$ in the sub-figure for $G^{(i)}$ with $H \subseteq K$ we have $T_{H} \subseteq T_{K}$. Let us note that, by the construction of Figure 1 , if the sub-figure for $G^{(i)}$, $1 \leq i \leq n$, is complete then $T^{(i)}$ is extendable to $G^{(i-1)}$ and $T_{1, l}^{(i)}$ is extendable to $G_{1, \ell}^{(i-1)}, 0 \leq \ell \leq i$.

DEFINITION 6. The row for $G^{(i)}, 1 \leq i \leq n$, is complete if there exist subgroups $T^{(i)}, T_{1, \ell}^{(i)}, 0 \leq \ell \leq i$, such that

$$
\begin{aligned}
& \text { (i) } T^{(i)} \supseteq T_{1,0}^{(i)} \supseteq T_{1,1}^{(i)} \supseteq \cdots \supseteq T_{1, i}^{(i)} \supseteq<0>, \\
& \text { (ii) } G^{(i)}=S^{(i)} \oplus T^{(i)}, G_{1, \ell}^{(i)}=S_{1, \ell}^{(i)} \oplus T_{1, \ell}^{(i)}, 0 \leq \ell \leq i, \\
& \text { (iii) } T^{(i)} \text { is extendable to } G^{(i-1)} \text { and } T_{1, \ell}^{(i)} \text { is extendable to } G_{1, \ell}^{(i-1)}, 0 \leq \ell \leq i
\end{aligned}
$$


We will say that the sub-figure (row) for $G^{(i)}$ can be completed if we can prove the existance of the subgroups $\mathrm{T}_{H}\left(\mathrm{~T}^{(i)}, \mathrm{T}_{1, l}^{(i)}\right.$ discussed in the definition for "The subfigure (row) for $G^{(i)}$ is complete."

PROPOSITION 1. Let $G$ be a finite abelian $p$-group and let $G=G^{(0)}=S \oplus A \supset G^{(1)}=S^{(1)} \oplus A^{(1)} \supset \ldots \supset G^{(n)}=S^{(n)} \oplus A^{(n)} \supset<0>$ be a composition series for $G$ with $S=S^{(0)} \supseteq S^{(1)} \supseteq \ldots \supseteq S^{(n)} \supseteq\langle 0\rangle$ subgroups of $G$ and $A=A^{(0)} \supseteq A^{(1)} \supseteq \ldots \supseteq A^{(n)} \supseteq\{0\} Z$-sets. The following statements are true for $0 \leq i \leq n-1,0 \leq i^{\prime} \leq n$ :
(a) $\left[G_{1, i}^{(i)}: G_{1, i+1}^{(i+1)}\right] \leq p$
(b) $\left[G_{1, \ell}^{(i)}: G_{1, \ell}^{(i+1)}\right] \leq p, 0 \leq \ell \leq i+1$
(c) $\left[G_{1, \ell^{\prime}+1}^{(i)}: G_{1, \ell^{\prime}+1}^{(i+1)}\right] \leq\left[G_{1, \ell^{\prime}}^{(i)}: G_{1, \ell}^{(i+1)}\right], 0 \leq \ell^{\prime} \leq i$
(d) $\left[G_{1, \ell}^{\left(i^{\prime}\right)}: G_{1, \ell^{\prime}+1}^{\left(i^{\prime}\right)}\right] \leq p, 0 \leq \ell^{\prime} \leq i^{\prime}$.
(e) $\left[G_{1, \ell^{\prime}}^{(i+1)}: G_{1, \ell^{\prime}+1}^{(i+1)}\right] \leq\left[G_{1, \ell^{\prime}}^{(i)}: G_{1, \ell^{\prime}+1}^{(i)}\right], 0 \leq \ell^{\prime} \leq i$.
(f) $\left[G_{2, \ell}^{(i)}: G_{2, \ell}^{(i+1)}\right] \leq\left[G_{1, \ell}^{(i)}: G_{1, \ell}^{(i+1)}\right], 0 \leq \ell \leq i+1$

PROOF. Let $G^{(i)}=\underset{k=1}{\mathbf{r}}\left\langle\mathrm{g}_{k}>\right.$. Since $\left[G^{(i)}: G^{(i+)}\right]=p$ we have $G^{(i+1)}=\left\langle g_{1}\right\rangle \oplus\left\langle g_{2}\right\rangle \oplus \ldots \oplus\left\langle p_{j}\right\rangle \oplus \ldots \oplus\left\langle g_{k}\right\rangle$ for some $j, 1 \leq j \leq r$.

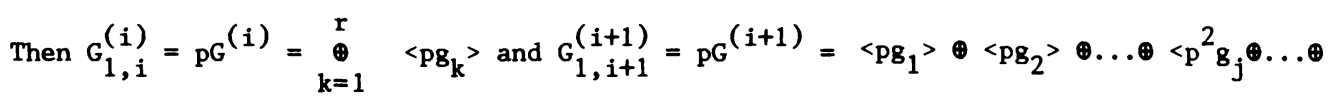
$<\mathbf{p g}_{\mathrm{k}}>$. If $o\left(g_{j}\right)=p$, then $G_{1, i}^{(i)}=G_{1, i+1}^{(i+1)}$. If $o\left(g_{j}\right)>p$, then $\left[G_{1, i}^{(i)}: G_{1, i+1}^{(i+1)}\right]=p$.

This proves (a).

Each of properties (b) through (f) can be deduced from Lemma 4 by choosing $U, U_{1}$, and $K$ appropriately as follows:
(b) $U=G^{(i)}, U_{1}=G^{(i+1)}, K=G_{1, \ell}^{(\ell)}, 0 \leq \ell \leq i+1$.
(c) $U=G_{1, \ell^{\prime}}^{(i)}, U_{1}=G_{1, \ell^{\prime}}^{(i+1)}, K=G_{1, \ell^{\prime}+1}^{\left(\ell^{\prime}+1\right)}, 0 \leqq \ell^{\prime} \leqq i$. 
(d) $U=G_{1, \ell^{\prime}}^{\left(\ell^{\prime}\right)}, U_{1}=G_{1, \ell^{\prime}+1}^{\left(\ell^{\prime}+1\right)}, K=G^{\left(i^{\prime}\right)}, 0 \leqq \ell \leqq i^{\prime}$.

(e) $U=G_{1, \ell^{\prime}}^{(i)}, U_{1}=G_{1, \ell^{\prime}+1}^{(i)}, K=G^{(i+1)}, 0 \leq \ell^{\prime} \leq i$.

(f) $U=G_{1, \ell}^{(i)}, U_{1}=G_{1, \ell}^{(i+1)}, K=G_{2, \ell}^{(\ell)}, 0 \leqq \ell \leqq i+1$.

Observe that $\left[G^{(i)}: G^{(i+1)}\right]=p$ implies that $p G^{(i)} \subseteq G^{(i+1)}$ and $p^{2} G^{(i)} \subseteq p G^{(i+1)} \subseteq G^{(i+2)}$. Thus $\left[G_{1, i}^{(i)}: G_{1, i}^{(i+1)}\right]=1,0 \leqq i \leqq n-1,\left[G_{2, i}^{(i)}: G_{2, i}^{(i+1)}\right]=1,0 \leq i \leq n-1$, and $\left[G_{2, i}^{(i+1)}: G_{2, i}^{(i+2)}\right]=1,0 \leq i \leq n-2$.

PROPOSITION 2. Let $G$ be a finite abelian $p$-group and let $G=G^{(0)}=S \oplus A \supset G^{(1)}=S^{(1)} \oplus A^{(1)} \supset \ldots G^{(n)}=S^{(n)} \oplus A^{(n)} \supset<0<$ be a composition series for $G$ with $\left.S=s^{(0)} \supseteq s^{(1)} \supseteq \ldots \supseteq s^{(n)} \supset<0\right\rangle$ subgroups of $G$ and $A=A^{(0)} \supseteq A^{(1)} \supseteq \ldots \supseteq A^{(n)} \supseteq\{0\}$ Z-sets. The following statements are true for $0 \leq i \leq n-1,0 \leq \ell \leq i, 0 \leq \ell^{\prime} \leq i-1$.

(a) If $\left[G_{1, l}^{(i)}: G_{1, l+1}^{(i+1)}\right]=1$ and $\left[G_{1, \ell}^{(i)}: G_{1, l}^{(i+1)}\right]=p$, then $\left[G_{2, l}^{(i)}: G_{2, l}^{(i+1)}\right]=1$.

(b) If $\left[G_{1, \ell^{\prime}+1}^{(i)}: G_{1, \ell^{\prime}+1}^{(i+1)}\right]=\left[G_{1, \ell^{\prime}+1}^{(i-1)}: G_{1, \ell^{\prime}+1}^{(i)}\right]=1$ and

$$
\left[G_{1, \ell^{\prime}}^{(i)}: G_{1, \ell^{\prime}}^{(i+1)}\right]=p \text {, then }\left[G_{1, \ell^{\prime}}^{(i-1)}: G_{1, \ell^{\prime}}^{(i)}\right]=1 \text {. }
$$

PROOF. We have $G_{2, l}^{(i)} \subseteq G_{1, \ell+1}^{(i)}=G_{1, \ell+1}^{(i)} \subseteq G^{(i+1)}$. Therefore $G_{2, l}^{(i)}=G_{2, \ell}^{(i)} \cap G^{(i+1)}$

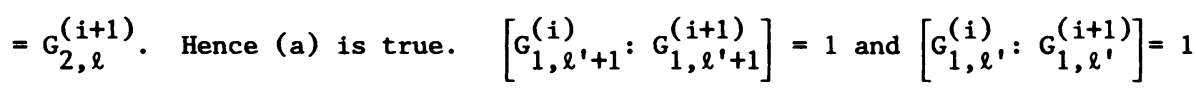
imply that $\left[G_{1, \ell^{\prime}:}^{(i)} G_{1, \ell^{\prime}+1}^{(i)}\right]=p$ and $\left[G_{1, \ell^{\prime}}^{(i+1)}: G_{1, \ell^{\prime}+1}^{(i+1)}\right]=1$. By (d) and (e) of Proposition 1 we have that $\left[G_{1, \ell^{\prime}}^{(i-1)}: G_{1, \ell^{\prime}+1}^{(i+1)}\right]=$ p. Consequent $1 y\left[G_{1, \ell^{\prime}}^{(i-1)}: G_{1, \ell^{\prime}}^{(i)}\right]=1$.

We can eliminate from consideration several combinations of indices in Figure 1 since by Proposition 2 it is impossible for them to occur.

LEMMA 6 . Let $W, X, Y$ and $Z$ be subgroups with the following properties:

(i) $\mathrm{W} \subseteq \mathrm{X}, \mathrm{Y} \subseteq \mathrm{X}, \mathrm{Z}=\mathrm{W} \cap \mathrm{Y}$

(ii) $[\mathrm{W}: Z]=[\mathrm{X}: \mathrm{Y}]=\mathrm{p}$

(iii) $X=S_{X} \oplus A_{X}, W=S_{W} \oplus A_{W}=S_{W} \oplus T_{W}, Y=S_{X} \oplus A_{Y}=S_{X} \oplus T_{Y}$, where $S_{W}, S_{X}, T_{W}, T_{Y}$ are subgroups with $S_{W} \subset S_{X}$ and $A_{W}, A_{Y}, A_{X}$ are Z-sets with $A_{W} \subseteq A_{X}, A_{Y} \subseteq A_{X}$.

(iv) $\mathrm{Z}=\mathrm{S}_{\mathrm{W}} \oplus \mathrm{T}_{\mathrm{Z}}$ with $\mathrm{T}_{\mathrm{Z}} \subseteq \mathrm{T}_{\mathrm{W}}$ and $\mathrm{T}_{\mathrm{Z}} \subseteq \mathrm{T}_{\mathrm{Y}}$. 
Then $\mathrm{X}=\mathrm{S}_{\mathrm{X}} \oplus \mathrm{T}_{\mathrm{X}}$, where $\mathrm{T}_{\mathrm{X}}=\mathrm{T}_{\mathrm{W}}+\mathrm{T}_{\mathrm{Y}}$.

PROOF. By Lemma 1 we have $Z=W \cap Y=\left(S_{W} \cap S, \cap\left(A_{W} \cap A_{Y}\right)=S_{W} \oplus\left(A_{W} \cap A_{Y}\right)\right.$. The following diagram illustrates the relations between the subgroups $W, X, Y$, and $Z$.

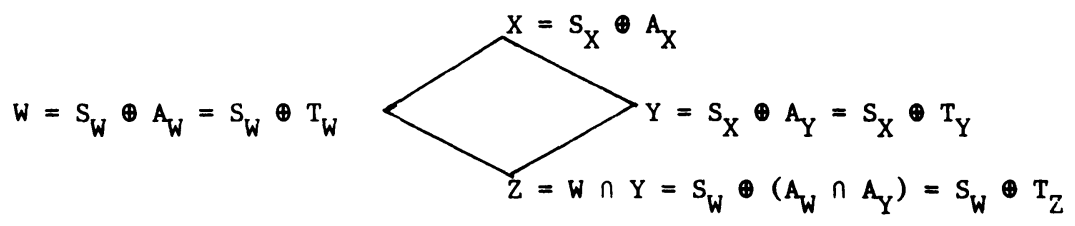

We will first show that $X=W+Y$. We have $Y \subseteq W+Y \subseteq X$ and $[W: Y]=p$. Since $[W: Z]=p$ we must have $X=W+Y=S_{X}+\left(T_{W}+T_{Y}\right)$.

We will complete the proof by showing that $S_{X} \cap\left(T_{W}+T_{Y}\right)=\langle 0\rangle$. Let

$$
s_{X}=t_{W}+t_{Y}, s_{X} \in S_{X}, t_{W} \in T_{W}, t_{Y} \in T_{Y}
$$

We can write $t_{W}=s_{W}+a_{W}, t_{Y}=s_{X}^{\prime}+a_{Y}, s_{W} \in s_{W}, s_{X}^{\prime} \in s_{X}, a_{W} \in A_{W}, a_{Y} \in A_{Y}$.

Thus (5.2) becomes $s_{X}=s_{W}+a_{W}+s_{X}^{\prime}+a_{Y}$ and we have $s_{X}-s_{X}^{\prime}=s_{W}$ and

$-a_{W}=a_{Y} \in A_{W} \cap A_{Y} \subseteq Z$. Consequently we can write $a_{W}=s_{W}^{\prime}+t_{Z}, s_{W}^{\prime} \in S_{W}, t_{Z} \in T_{Z}$. But then $t_{W}=s_{W}+s_{W}^{\prime}+t_{Z}$. Since $T_{Z} \subseteq T_{W}$ and $T_{W} \cap S_{W}=\langle 0\rangle$ we must have $s_{W}+s_{W}^{\prime}=0$. Similarly, $t_{Y}=s_{X}^{\prime}-s_{W}^{\prime}-t_{Z}$ so that $s_{X}^{\prime}-s_{W}^{\prime}=0$. Hence $s_{X}=s_{W}+s_{W}^{\prime}-s_{W}^{\prime}-s_{X}^{\prime}=0$.

THEOREM 5. Let $G$ be a finite abelian $p$-group of exponent $p^{k}, k \geqq 1$, and let

$$
G=G^{(0)}=S \oplus A \supset G^{(1)}=S^{(1)} \oplus A^{(1)} \supset \ldots G^{(n)}=S^{(n)} \oplus A^{(n)} \supset\langle 0\rangle
$$

be a series for $G$ with $S=s^{(0)} \supseteq s^{(1)} \supseteq \ldots \underline{\rho} s^{(n)} \supseteq\langle 0\rangle$ subgroups of $G$ and $A=A^{(0)} \supseteq A^{(1)} \supseteq \underline{\ldots} . \underline{A^{(n)}} \supseteq\{0\}$ z-sets. If $p^{2} A=\{0\}$ then the series (5.3) admits replacements.

PROOF. By Lemma 3 we can assume that (5.3) is a composition series for G. Note that for $0 \leq i \leq n, 0 \leq \ell \leq i, j \geq 2, h \geq 1$,

$$
A_{j, l}^{(i)}=A^{(i)} \cap p^{j} A^{(l)} \subseteq P^{2} A=\{0\} .
$$

and

$$
p^{h} A_{1, l}^{(i)} \subseteq p^{h+1} A^{(l)} \subseteq p^{2} A=\{0\} .
$$

Thus we have $G_{j, l}^{(i)}=s_{j, l}^{(i)}$ and $p^{h} G_{1, l}^{(i)}=p^{h} s_{1, l}^{(i)}, 0 \leq i \leq n, 0 \leq \ell \leq i, j \geqq 2, h \geq 1$. Consequently we must have $T_{j, l}^{(i)}=T_{h, l, l}^{(i)}=\langle 0>, 0 \leq i \leq n, 0 \leq \ell \leq i, j \geq 2, h \geq 1$.

We use a "backward induction" on $i$ to show that the sub-figure for each $G^{(i)}$, $0 \leqq i \leqq n$, can be completed.

For $i=n,\left|G^{(n)}\right|=p$ implies that $G^{(n)}$ is cyclic of forder $p$. Thus the subfigure for $G^{(n)}$ is trivially complete. 
Now assume the sub-figure for $G^{(i+1)}$ is complete. In view of the preceeding comments on the subgroups $T_{j, l}^{(i)}, T_{h, 1, \ell}^{(i)}, 0 \leqq \ell \leqq i, j \geqq 2, h \geqq 1$, if we can complete the row for $G^{(i)}$ in such a way that $T_{1, l}^{(i+1)} \subseteq T_{1, \ell}^{(i)}, 0 \leq \ell \leq i, T^{(i+1)} \subseteq T^{(i)}$, then the sub-figure for $G^{(i)}$ will be complete as well.

By Lemma 3 we must consider two cases, (1) $A^{(i)}=A^{(i+1)}$, and (2) $s^{(i)}=S^{(i+1)}$.

Case (1). By hypothesis, the sub-figure for $\mathrm{G}^{(i+1)}$ is complete so that there exist subgroups $T^{(i+1)} \supseteq T_{1,0}^{(i+1)} \supseteq T_{1,1}^{(i+1)} \supseteq \ldots \supseteq T_{1, i+1}^{(i+1)}$ such that

$$
G^{(i+1)}=S^{(i+1)} \oplus T^{(i+1)}, G_{1, \ell}^{(i+1)}=S_{1, \ell}^{(i+1)} \oplus T_{1, \ell}^{(i+1)}, 0 \leq \ell \leq i+1 \text {, and } T^{(i+1)} \text { is }
$$

extendable to $G^{(i)}, T_{1, \ell}^{(i+1)}$ is extendable to $G_{1, \ell}^{(i)}, 0 \leqq \ell \leqq i$. Setting $H=G^{(i)}$, $K=p G^{(\ell)}, 0 \leq \ell \leq i$, in Lemma 1 , we conclude that $A_{1, \ell}^{(i)}=A_{1, \ell}^{(i+1)}, 0 \leq \ell \leq i$. Applying Lemma 5 with $G=G_{1, \ell}^{(i)}, G^{\prime}=G_{1, \ell}^{(i+1)}, T=T_{1, \ell}^{(i+1)}, 0 \leqq \ell \leqq i$, we have $G_{1, \ell}^{(i)}=S_{1, \ell}^{(i)} \oplus T_{1, \ell}^{(i+1)}, 0 \leqq \ell \leqq i$. Again applying Lemma 5, this time with $G=G^{(i)}$, $G^{\prime}=G^{(i+1)}, T=T^{(i+1)}$, we have $G^{(i)}=S^{(i)} \oplus T^{(i+1)}$. Thus we can complete the subfigure for $G^{(i)}$ by choosing $T_{1, \ell}^{(i)}=T_{1, l}^{(i+1)}, 0 \leqq \ell \leqq i, T^{(i)}=T^{(i+1)}$.

Case (2). We have $\left[G_{1, \ell}^{(i)}: G_{1, \ell}^{(i+1)}\right] \leqq p, 0 \leqq \ell \leq i$, by (b) of Proposition 1 . If $\left[G_{1, \ell}^{(i)}: G_{1, \ell}^{(i+1)}\right]=1,0 \leqq \ell \leqq i$, we can complete the sub-figure for $G^{(i)}$ by choosing $T_{1, \ell}^{(i)}=T_{1, \ell}^{(i+1)}, 0 \leqq \ell \leqq i$, and extending $T^{(i+1)}$ to $G^{(i)} \cdot T^{(i+1)}$ is extendable to $G^{(i)}$ by the hypothesis that the sub-figure for $\mathrm{G}^{(i+1)}$ is complete).

Now suppose there exists $\ell_{0}$ such that

$$
\left[G_{1, \ell}^{(i)}: G_{1, \ell}^{(i+1)}\right]=\left\{\begin{array}{l}
1 \text { for } \ell_{0}<\ell \leqq i \\
p \text { for } 0 \leqq \ell \leqq \ell
\end{array}\right.
$$

This situation is illustrated in Figure 2, where, for simplicity, we have omitted the subgroups $G_{j, \ell}^{(r)}$ and $G_{h, 1, \ell}^{(r)}$ since $T_{j, \ell}^{(r)}=T_{h, 1, \ell}^{(r)}=\langle 0>, 0 \leq \ell \leq n, 1 \leq h \leq k-2$,

$2 \leq j \leq k-1, r=i+1, i, i-1$. The numbers 1 and $p$ in Figure 2 represent indices.

We can choose $\mathrm{T}_{1, \ell}^{(i)}=\mathrm{T}_{1, \ell}^{(i+1)}$ for $\ell_{0}<\ell \leqq i$. As remarked previous $1 \mathrm{y}$, the hypothesis that the sub-figure for $G^{(i+1)}$ is complete implies that $T_{1, l_{0}}^{(i+1)}$ can be extended to $G_{1, l}^{(i)}$. This extension is indicated in Figure 2 by a single arrow.

Setting $X=G_{1, \ell}^{(i)}, Y=G_{1, \ell}^{(i+1)}, W=G_{1, \ell+1}^{(i)}, 0 \leq \ell \leq \ell,-1$, we have

$Z=W \cap Y=G_{1, \ell+1}^{(i+1)}, 0 \leqq \ell \leqq \ell_{0}-1$, so that $W, X, Y, Z$ satisfy the conditions of Lemma 6 . 
Thus we can apply Lemma 6 to obtain $G_{1, l}^{(i)}=S_{1, l}^{(i+1)} \oplus T_{1, l}^{(i)}$, where $T_{1, l}^{(i)}=T_{1, l+1}^{(i)}+T_{1, l}^{(i+1)}$, $0 \leq \ell \leq \ell_{0}-1$. We can again apply Lemma 6 , taking $X=G^{(i)}, W=G_{1,0}^{(i)}, Y=G^{(i+1)}$, $Z=W \cap Y=G_{1,0}^{(i+1)}$, to obtain $G^{(i)}=S^{(i+1)} \oplus T^{(i)}$, where $T^{(i)}=T_{1,0}^{(i)}+T^{(i+1)}$. These sums are indicated in Figure 2 by double arrows. Clearly, $T^{(i)} \supseteq T_{1,0}^{(i)} \supseteq \ldots \supseteq T_{1, l}^{(i)}$ and $T_{1, \ell}^{(i+1)} \subset T_{1, \ell}^{(i)}, 0 \leq \ell \leq i$. Thus the sub-figure for $G^{(i)}$ is complete.

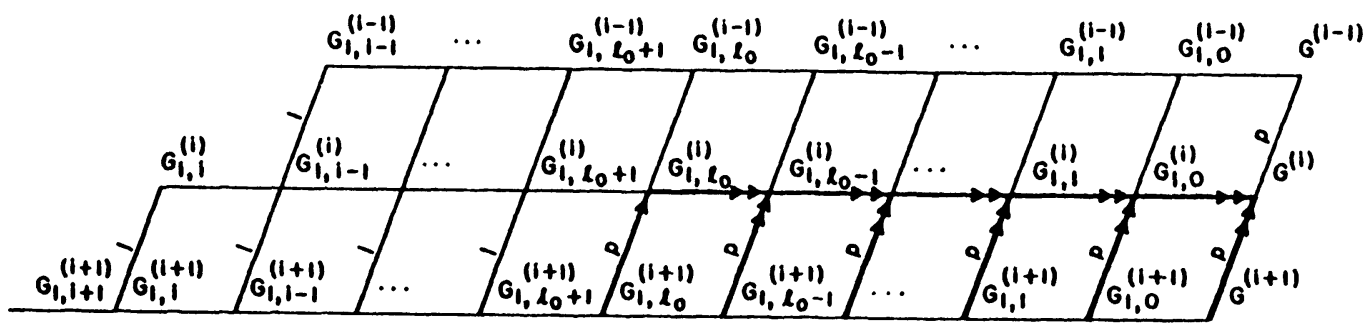

COROLLARY 1. If $G$ is a finite abelian $p$-group of exponent less than or equal to $\mathrm{p}^{2}$ then $G$ admits replacement.

$$
\text { PROOF. Let } G=G^{(0)}=S \oplus A \supset G^{(1)}=S^{(1)} \oplus A^{(1)} \supset \ldots \supset G^{(n)}=S^{(n)} \oplus A^{(n)} \supset\langle 0\rangle \quad(5.4)
$$

be a series for $G$ with $S=s^{(0)} \supseteq s^{(1)} \supseteq \ldots \supseteq s^{(n)} \supseteq\langle 0\rangle$ subgroups of $G$ and $A=A^{(0)} \supseteq A^{(1)} \supseteq \ldots \supseteq A^{(n)} \supseteq\{0\}$ Z-sets. We have $p^{2} A=\{0\}$ since by hypothesis $p^{2} G=\langle 0\rangle$. By Theorem 5 the series (5.4) admits replacements. Hence $G$ admits replacement.

6. RELATION TO A VARIATION OF A METHOD OF A.D. SANDS.

Our terminology will be the same as in [3] when referring to factorizations which are obtained by the variation of Sands' method.

The following Proposition can be readily verified.

PROPOSITION 3. Let $G=k_{1} \supset k_{2} \supset \ldots \supset k_{n} \supset<0>$ be a series for $G$ with coset representatives $H_{i}, 1 \leq i \leq n, K_{n}=H_{n}$. If $H_{i} \oplus H_{i+2} \oplus \ldots$ is a subgroup (Z-set) then $\mathrm{H}_{\mathrm{i}+2} \oplus \mathrm{H}_{\mathrm{i}+4} \oplus \ldots$ is also a subgroup (Z-set).

THEOREM 6. Let $G$ be a finite abelian group which admits replacement and let

$$
\mathrm{G}=\mathrm{K}_{1} \supset \mathrm{K}_{2} \supset \ldots \supset \mathrm{K}_{\mathrm{n}} \supset\langle 0\rangle
$$

be a series for $G$. If $G=A \oplus B$ is a $Z$-factorization of $G$ arising from (6.1) then there exist subgroups $S, T$ such that the factorization $G=S \oplus T$ arises from the series (6.1).

PROOF. We will assume that $\mathrm{n}$ is odd since the proof for $\mathrm{n}$ even is similar. We will proceed by induction on the order of the group $G$.

If $|G|=p$, then $G=G \oplus\langle 0\rangle$ is the only $Z$-factorization of $G$. Thus in any series from which this factorization arises we must have $G=K_{1}=S, T=K_{i}=\langle 0\rangle, i \geq 2$. 
Assume the theorem is true for groups of order less than $G$. Let $G=A \oplus B$ be a Z-factorization of $G$ arising from (6.1). By Lemma 5 of [3] we may assume

$$
\begin{aligned}
& A=\mathrm{H}_{1} \oplus \mathrm{H}_{3} \oplus \mathrm{H}_{5} \oplus \ldots \oplus \mathrm{H}_{\mathrm{n}}, \\
& \mathrm{B}=\mathrm{H}_{2} \oplus \mathrm{H}_{4} \oplus \mathrm{H}_{6} \oplus \ldots \oplus \mathrm{H}_{\mathrm{n}-1},
\end{aligned}
$$

where $0 \in H_{i}, 1 \leq i \leq n$.

We have the $\mathrm{H}_{3}+\mathrm{H}_{5}+\ldots+\mathrm{H}_{n}$ is a Z-set by Proposition 3 .

Thus

$$
K_{2}=\left(H_{3} \oplus H_{5} \oplus \ldots \oplus H_{n}\right) \oplus\left(H_{2} \oplus H_{4} \oplus \ldots \oplus H_{n-1}\right)
$$

is a Z-factorization of $\mathrm{K}_{2}$ arising from the series

$$
k_{2} \supset k_{3} \supset \ldots \supset k_{n} \supset<0>
$$

$\left|K_{2}\right|<|G|$ implies, by the induction hypothesis, that there exist subgroups, $S^{\prime}, T^{\prime}$ such that the factorization $K_{2}=S^{\prime} \oplus T^{\prime}$ arises from the series (6.2). Thus, by Lemma 5 [3] there exist transversals $H_{i}^{\prime}, 2 \leqq i \leqq n$, such that

$$
\begin{aligned}
& \mathrm{S}^{\prime}=\mathrm{H}_{2}^{\prime} \oplus \mathrm{H}_{4}^{\prime} \oplus \ldots \oplus \mathrm{H}_{\mathrm{n}-1}^{\prime}, \\
& \mathrm{T}^{\prime}=\mathrm{H}_{3}^{\prime} \oplus \mathrm{H}_{5}^{\prime} \oplus \ldots \oplus \mathrm{H}_{\mathrm{n}^{\prime}}^{\prime},
\end{aligned}
$$

where $0 \in H_{i}^{\prime}, 2 \leqq i \leq n$.

Note that $\mathrm{K}_{\mathrm{n}}=\mathrm{H}_{\mathrm{n}}=\mathrm{H}_{\mathrm{n}}^{\prime}$ and

$$
K_{i}=H_{i}^{\prime} \oplus K_{i+1}=H_{i} \oplus K_{i+1}, 2 \leqq i \leqq n-1
$$

since both $\mathrm{H}_{i}$ and $\mathrm{H}_{i}^{\prime}$ are coset representatives for $\mathrm{K}_{i}$ modulo $\mathrm{K}_{i+1}, 2 \leq i \leq n$. Using (6.3) successively, starting with $i=n-1$, we see that we can choose $H_{1}, H_{3}, H_{5}, \ldots$, $H_{n}, H_{2}^{\prime}, H_{4}^{\prime}, H_{6}^{\prime}, \ldots, H_{n-1}^{\prime}$ as coset representatives for the series (6.1) to obtain the factorization

$$
G=\left(H_{1} \oplus H_{3} \oplus \ldots \oplus H_{n}\right) \oplus\left(H_{2}^{\prime} \oplus H_{4}^{\prime} \oplus \ldots \oplus H_{n-1}^{\prime}\right)=A \oplus S^{\prime}
$$

By Proposition 3, $\mathrm{H}_{i} \oplus \mathrm{H}_{\mathrm{i}+2} \oplus \ldots \oplus \mathrm{H}_{\mathrm{n}}$ is a Z-set, $\mathrm{i}=1,3, \ldots \mathrm{n}$, and $\mathrm{H}_{i}^{\prime} \oplus \mathrm{H}_{\mathrm{i}+2}^{\prime} \oplus \ldots \oplus$ $H_{n-1}^{\prime}$ is a subgroup, $i=2,4, \ldots, n-1$. Set

$$
\begin{aligned}
& S^{(i)}=H_{i}^{\prime} \oplus H_{i+2}^{\prime} \oplus \ldots \oplus H_{n-1}^{\prime}, i=2,4, \ldots, n-3 \\
& s^{(n-1)}=H_{n-1}^{\prime} \\
& A^{(i)}=H_{i} \oplus H_{i+2} \oplus \ldots \oplus H_{n}, i=1,3, \ldots, n-2 \\
& A^{(n)}=H_{n}=K_{n} .
\end{aligned}
$$


Then the series $(6.1)$ can be written as

$G=K_{1}=S^{(2)} \oplus A^{(1)} \supset K_{2}=S^{(2)} \oplus A^{(3)} \supset K_{3}=S^{(4)} \oplus A^{(3)} \supset \ldots \supset K_{n-1}=S^{(n-1)} \oplus A(n) \supset K_{n}=A(n) \supset\langle 0\rangle$

where $S^{(2)}=S^{\prime}$ and $A^{(1)}=A$. In general,

$$
K_{i}=\left\{\begin{array}{l}
S^{(i)} \oplus A^{(i+1)} \text { for } i \text { even, } 2 \leq i \leq n-1 \\
S^{(i+1)} \oplus A^{(i)} \text { for } i \text { odd, } 1 \leq i \leq n-2, K_{n}=A^{(n)}=H_{n} .
\end{array}\right.
$$

By hypothesis $G$ admits replacement. Thus there exist subgroups $T^{(1)} \supseteq T^{(2)} \supseteq \ldots \supseteq T^{(n)}$ such that

$$
k_{i}=\left\{\begin{array}{l}
s^{(i)} \oplus T^{(i)} \text { for } i \text { even, } 2 \leq i \leq n-1, \\
S^{(i+1)} \oplus T^{(i)} \text { for } i \text { odd, } 1 \leq i \leq n-2, K_{n}=T^{(n)}=H_{n}=A^{(n)} .
\end{array}\right.
$$

Define $H_{n}^{\prime \prime}=T^{(n)}=A^{(n)}$. We have

$$
K_{n-1}=S^{(n-1)} \oplus T^{(n-1)}=S^{(n-1)} \oplus A^{(n)}=S^{(n-1)} \oplus T^{(n)}
$$

so that $\left|T^{(n-1)}\right|=\left|T^{(n)}\right|$. But $T^{(n)} \subseteq T^{(n-1)}$. Therefore $T^{(n-1)}=T^{(n)}$.

Next,

$$
K_{n-2}=S^{(n-1)} \oplus T^{(n-2)}, T^{(n-1)} \subseteq T^{(n-2)}
$$

If we choose $\mathrm{H}_{n-2}^{\prime \prime}$ a set of coset representatives for $T^{(n-2)}$ modulo $T^{(n-1)}$ we have $T^{(n-2)}=H_{n-2}^{\prime \prime} \oplus T^{(n-1)}=H_{n-2}^{\prime \prime} \oplus T^{(n)}=H_{n-2}^{\prime \prime}+H_{n}^{\prime \prime}$, and $K_{n-2}=S^{(n-1)} \oplus H_{n-2}^{\prime \prime} \oplus T^{(n)}$

$=H_{n-2}^{\prime \prime} \oplus K_{n-1}$. Thus $H_{n-2}^{\prime \prime}$ is also a set of coset representatives for $K_{n-2}$ modulo $K_{n-1}$.

$$
K_{n-3}=S^{(n-3)} \oplus T^{(n-3)}=S^{(n-3)} \oplus A^{(n-2)} \text { and }
$$

$K_{n-2}=S^{(n-1)} \oplus T^{(n-2)}=S^{(n-1)} \oplus A^{(n-2)}$ imply that $\left|T^{(n-3)}\right|=\left|A^{(n-2)}\right|=\left|T^{(n-2)}\right|$.

But $T^{(n-2)} \subset T^{(n-3)}$. Hence we have that $T^{(n-2)}=T^{(n-3)}$ and

$$
K_{n-3}=S^{(n-3)} \oplus T^{(n-2)}=\left(H_{n-3}^{\prime} \oplus H_{n-1}^{\prime}\right) \oplus T^{(n-2)}=H_{n-3}^{\prime} \oplus K_{n-2} \text {. }
$$

In general, given $T^{(i)}$ for $i$ odd we have $T^{(i-1)}=T^{(i)}$ and $T^{(i-2)}=H_{i-2}^{\prime \prime} \oplus T^{(i)}$ so that the factorizations of $k_{i}, 1 \leq i \leq n$, are as follows:

$$
\begin{aligned}
& K_{n}=T^{(n)}=H_{n}^{\prime \prime}=H_{n} \\
& K_{n-1}=S^{(n-1)} \oplus T^{(n)}=H_{n-1}^{\prime} \oplus H_{n}^{\prime \prime} \\
& K_{n-2}=S^{(n-1)} \oplus T^{(n-2)}=H_{n-1}^{\prime} \oplus H_{n-2}^{\prime \prime} \oplus T^{(n)}=H_{n-1}^{\prime} \oplus\left(H_{n-2}^{\prime \prime} \oplus H_{n}^{\prime \prime}\right)
\end{aligned}
$$




$$
\begin{aligned}
& K_{n-3}=S^{(n-3)} \oplus T^{(n-2)}=\left(H_{n-3}^{\prime} \oplus H_{n-1}^{\prime}\right) \oplus\left(H_{n-2}^{\prime \prime} \oplus H_{n}^{\prime \prime}\right) \\
& K_{n-4}=S^{(n-3)} \oplus T^{(n-4)}=S^{(n-3)} \oplus H_{n-4}^{\prime \prime} \oplus T^{(n-2)}=\left(H_{n-3}^{\prime} \oplus H_{n-1}^{\prime}\right)+\left(H_{n-4}^{\prime \prime} \oplus H_{n-2}^{\prime \prime} \oplus H_{n}^{\prime \prime}\right)
\end{aligned}
$$$$
\mathrm{K}_{3}=\mathrm{S}^{(4)} \oplus \mathrm{T}^{(3)}=\mathrm{S}^{(4)} \oplus \mathrm{H}_{3}^{\prime \prime} \oplus \mathrm{T}^{(5)}=\left(\mathrm{H}_{4}^{\prime} \oplus \mathrm{H}_{6}^{\prime} \oplus \ldots \oplus \mathrm{H}_{\mathrm{n}-1}^{\prime}\right)+\left(\mathrm{H}_{3}^{\prime \prime} \oplus \mathrm{H}_{5}^{\prime \prime} \oplus \ldots \oplus \mathrm{H}_{\mathrm{n}}^{\prime \prime}\right)
$$$$
\mathrm{K}_{2}=\mathrm{S}^{(2)} \oplus \mathrm{T}^{(3)}=\left(\mathrm{H}_{2}^{\prime} \oplus \mathrm{H}_{4}^{\prime} \oplus \ldots \oplus \mathrm{H}_{\mathrm{n}-1}^{\prime}\right)+\left(\mathrm{H}_{3}^{\prime \prime} \oplus \mathrm{H}_{5}^{\prime \prime} \oplus \ldots \oplus \mathrm{H}_{\mathrm{n}}^{\prime \prime}\right)
$$$$
\mathrm{K}_{1}=\mathrm{S}^{(2)} \oplus \mathrm{T}^{(1)}=\mathrm{S}^{(2)} \oplus \mathrm{H}_{1}^{\prime \prime} \oplus \mathrm{T}^{(3)}=\left(\mathrm{H}_{2}^{\prime} \oplus \mathrm{H}_{4}^{\prime} \oplus \ldots \oplus \mathrm{H}_{\mathrm{n}-1}^{\prime}\right) \oplus\left(\mathrm{H}_{1}^{\prime \prime} \oplus \mathrm{H}_{3}^{\prime \prime} \oplus \ldots \oplus \mathrm{H}_{\mathrm{n}}^{\prime \prime}\right) \text {. }
$$

We can complete the proof by defining $\mathrm{S}=\mathrm{S}^{(2)}=\mathrm{H}_{2}^{\prime} \oplus \mathrm{H}_{4}^{\prime} \oplus \mathrm{H}_{6}^{\prime} \oplus \ldots \oplus \mathrm{H}_{\mathrm{n}-1}^{\prime}$ and $T=T^{(1)}=H_{1}^{\prime \prime} \oplus \mathrm{H}_{3}^{\prime \prime} \oplus \mathrm{H}_{5}^{\prime \prime} \oplus \ldots \oplus \mathrm{H}_{\mathrm{n}}^{\prime \prime}$ to obtain the factorization $\mathrm{G}=\mathrm{S} \oplus \mathrm{T}, \mathrm{S} \subseteq \mathrm{G}, \mathrm{T} \subseteq \mathrm{G}$, which arises from the series (6.1).

\section{REFERENCES}

1. SANDS, A.D., On the factorization of finite abelian groups I, Acta. Math. Acad. Sci. Hung. 8 $(1957), 65-86$.

2. HAJOS, G, Sur la factorisation des groupes abeliens, Casopis Pest. Math. Fys. 74(1950), 157-162.

3. OBAID, E.E., On a variation of Sands' method, Internat. J. Math. \& Math. Sci. 9(3)(1986), 597-604.

4. FUCHS, L., Abelian Groups, Pergamon Press, 1960.

5. OKUDA, C., The factorization of Abelian groups, doctoral dissertation, the Pennsylvania State University, University Park, PA, 1975. 


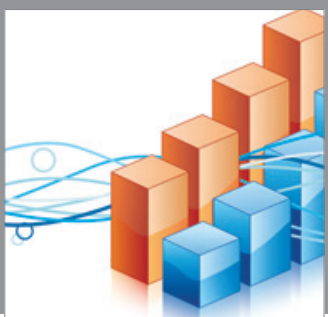

Advances in

Operations Research

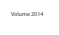

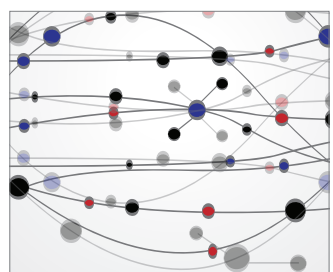

\section{The Scientific} World Journal
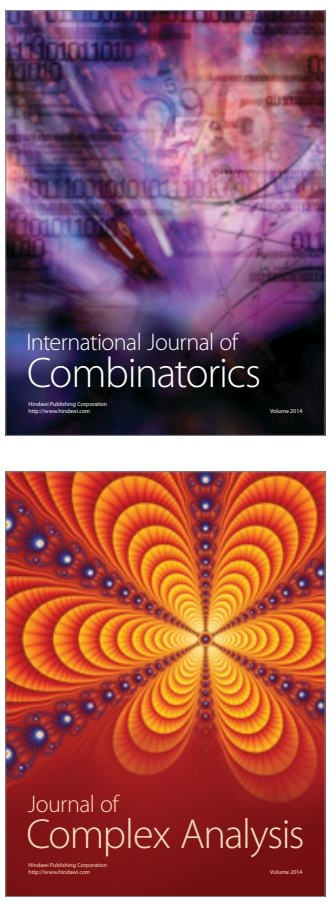

International Journal of

Mathematics and

Mathematical

Sciences
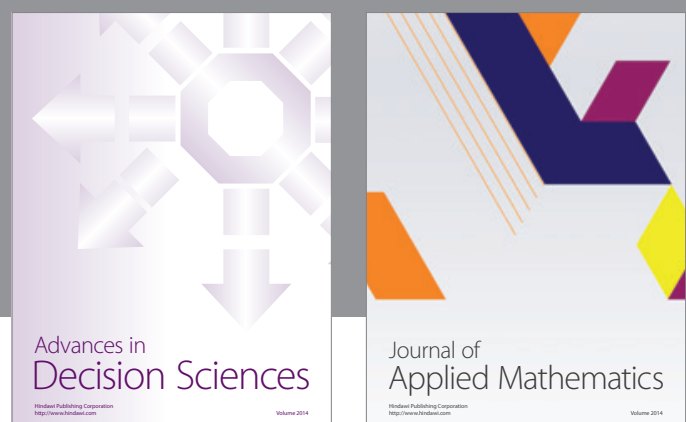

Journal of

Applied Mathematics
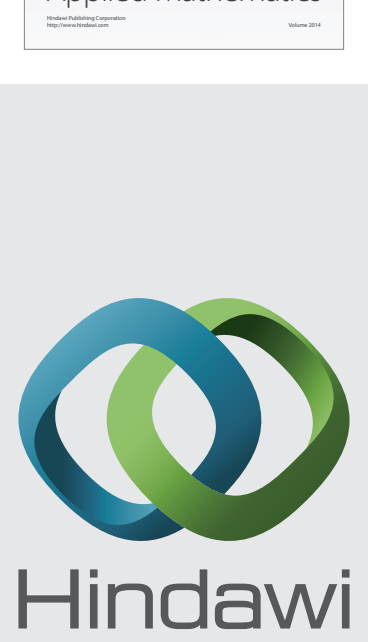

Submit your manuscripts at http://www.hindawi.com
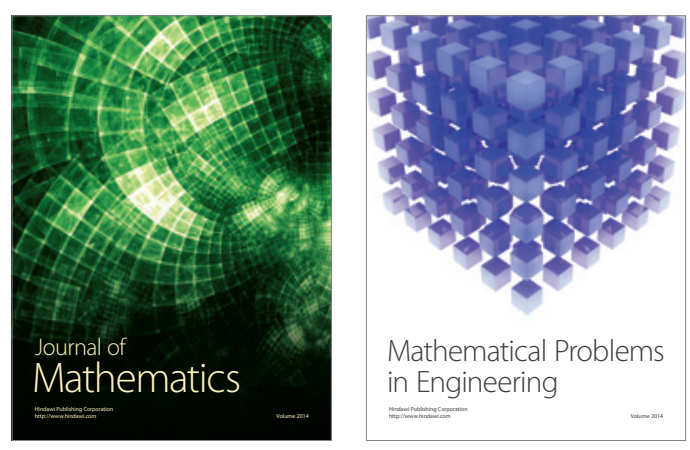

Mathematical Problems in Engineering
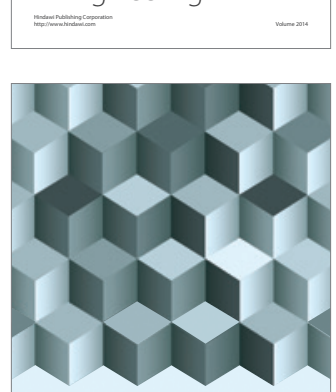

Journal of

Function Spaces
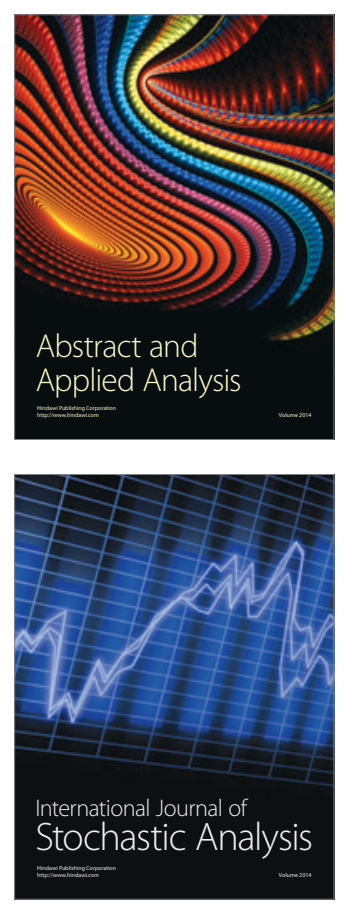

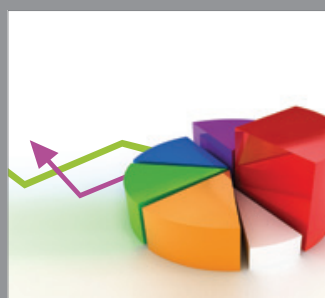

ournal of

Probability and Statistics

Promensencen
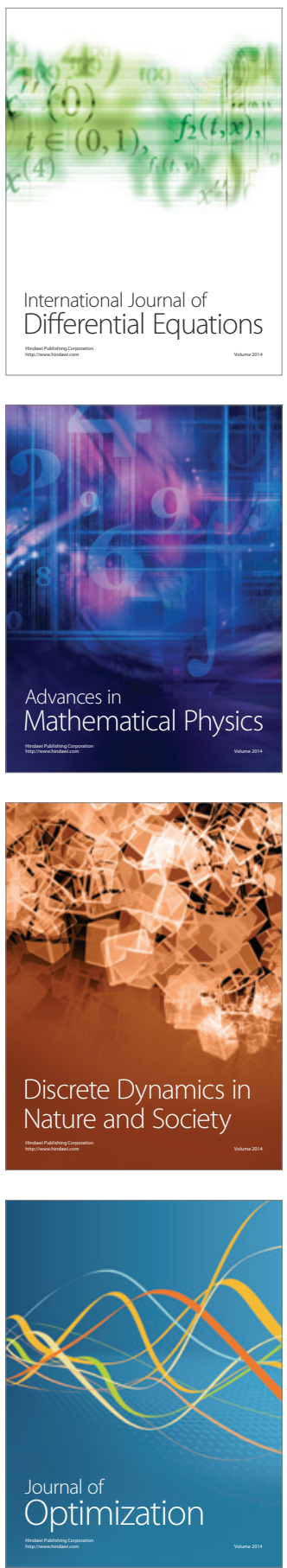